WP 22_12

\author{
Karim M. Abadir \\ Imperial College London, UK \\ The Rimini Centre for Economic Analysis (RCEA), Italy
}

Adriana Cornea

Imperial College London, UK

\title{
APPROXIMATING MOMENTS BY NONLINEAR TRANSFORMATIONS
}

Copyright belongs to the author. Small sections of the text, not exceeding three paragraphs, can be used provided proper acknowledgement is given.

The Rimini Centre for Economic Analysis (RCEA) was established in March 2007. RCEA is a private, nonprofit organization dedicated to independent research in Applied and Theoretical Economics and related fields. RCEA organizes seminars and workshops, sponsors a general interest journal The Review of Economic Analysis, and organizes a biennial conference: The Rimini Conference in Economics and Finance (RCEF) . The RCEA has a Canadian branch: The Rimini Centre for Economic Analysis in Canada (RCEACanada). Scientific work contributed by the RCEA Scholars is published in the RCEA Working Papers and Professional Report series.

The views expressed in this paper are those of the authors. No responsibility for them should be attributed to the Rimini Centre for Economic Analysis. 


\title{
Approximating moments by nonlinear transformations*
}

\author{
Karim M. Abadir and Adriana Cornea \\ Imperial College London
}

\begin{abstract}
We provide a methodology to calculate the expectation of a variate $x$ in terms of the moments of a transformation of $x$. Apart from the intrinsic interest in such a fundamental relation that relates the moments of a variate and its nonlinear transformations, our results can be used in practice to approximate $\mathrm{E}(x)$ by the low-order moments of a transformation which can be chosen to give a good approximation for $\mathrm{E}(x)$. To obtain an accurate evaluation of the remainder, we derive results for the bounding of functions of complex variables. Our results are useful, for example, in resampling applications like bootstrap confidence intervals for fat-tailed data. They are also useful in economics and finance in quantifying the effect of taking nonlinear transformations on moment conditions and on asset prices which are formulated as expectations.
\end{abstract}

\section{Introduction}

We provide a methodology to calculate the expectation of a variate $x \in \mathcal{X} \subseteq \mathbb{R}$ (with unknown density) in terms of the moments of an invertible transformation $y:=g^{-1}(x) \in$

*This research is supported by the ESRC grant RES062230790 and by the British Academy's $\mathrm{PDF} / 2009 / 370$. 
$\mathcal{Y} \subseteq \mathbb{R}$. This fundamental relation between the moments of $x$ and $y$ has, surprisingly, not been derived anywhere. Approximations to it have been used on an ad-hoc basis, typically through the leading terms of a Taylor expansion and without assessing either the goodness of such an expansion (as opposed to a more general one) or the evaluation of the remainder. In this paper, we provide an exact formula for general expansions linking these moments.

It is of interest to investigate such a fundamental relation. But apart from the intrinsic interest in it, it can be used in practice to approximate $\mathrm{E}(x)$ by the low-order moments of a transformation which can be chosen to give a good approximation for $\mathrm{E}(x)$. Such results are useful, for example, in resampling applications like bootstrap confidence intervals for fat-tailed data where the standard bootstrap fails because of the nonexistence of higher moments; e.g. see Athreya (1987), Knight (1989), Politis, Romano, and Wolf (1999). Such applications are substantial and beyond the scope of the current paper. Here, we focus on the mathematical derivations that underpin the approximation theory that can be used in these applications and in other ones like Taylor's (1986) assessment of the mean effect of a Box-Cox transformation. The potential for applications is not just statistical and econometric, but also in economics and finance where there is interest inter alia in quantifying the effect of taking nonlinear transformations on moment conditions (such as Euler's, arising from optimization) and on asset prices which are formulated as expectations; e.g. see Yu et al. (2006), Martin (2008), Backus, Chernov and Martin (2011). The effect of higher-order terms is important and needs to be quantified, as recent market turbulence has emphasized.

In Section 2, we introduce the expansion and the required results from complex analysis. These are needed for the bounding of functions of complex variables to obtain an accurate evaluation of the remainder of the approximation. In Section 3, we illustrate the expansion and the accuracy of the remainder's bound. Section 4 concludes. We follow the notation conventions proposed in Abadir and Magnus (2002). 


\section{Expansion of $\mathrm{E}(x)$ in terms of the moments of $y$}

Suppose for simplicity that we are interested in $\mathrm{E}(x)$ which is assumed to exist. We stress that the same approach will apply to the expansion of any moment of $x$, not just $\mathrm{E}(x)$. For example, for the expansion of $\mathrm{E}\left(x^{3}\right)$, we can replace $x$ by $z:=x^{3}$ and apply the same method below to $\mathrm{E}(z)$. This is also true of any function $z$ of $x$.

We propose two types of expansions, raw or centered. We start by explaining the idea behind the two expansions: (a) without recourse to complex variables; and (b) with the simple $g=\exp$, giving $x:=\exp (y) \in \mathbb{R}_{+}$. The two types of expansions are:

1. the raw (direct) expansion

$$
x=\sum_{j=0}^{k} \frac{y^{j}}{j !}+R_{k}
$$

2. the centered expansion

$$
x=\mathrm{e}^{\mathrm{E} y} \mathrm{e}^{y-\mathrm{E} y}=\mathrm{e}^{\mathrm{E} y} \sum_{j=0}^{k} \frac{(y-\mathrm{E} y)^{j}}{j !}+R_{k}^{\mathrm{c}} .
$$

The moments on the right-hand side exist because of the existence of $\mathrm{E}(x)$. The series obtained above is a special case of Teixeira's expansion which expands a function in terms of another; e.g. see Whittaker and Watson (1997, pp.131-133) or Abadir and Talmain (2005) for an application. In this illustration, $g$ was the exponential function but the derivations to follow would apply to other invertible functions. For more elaborate cases from the hypergeometric ${ }_{p} F_{q}$ family, see Abadir (1999) or Whittaker and Watson (1997), but one should bear in mind the convergence radius of the expansion used. For example, it would be more problematic to expand the logarithmic function, due to its slower (and conditional) convergence: $\log (\cdot)$ is of the hypergeometric ${ }_{2} F_{1}$ class, whereas $\exp (\cdot)$ is ${ }_{0} F_{0}$. Other examples include hyperbolic functions (which are members of the ${ }_{0} F_{1}$ class that 
converges even faster than $\left.{ }_{0} F_{0}\right)$ such as

$$
\begin{aligned}
& x:=\cosh (y)=\sum_{j=0}^{k} \frac{y^{2 j}}{(2 j) !}+R_{k}, \quad\left(\text { for } x \in \mathbb{R}_{+}\right), \\
& x:=\sinh (y)=\sum_{j=0}^{k} \frac{y^{2 j+1}}{(2 j+1) !}+R_{k}, \quad(\text { for } x \in \mathbb{R}),
\end{aligned}
$$

or trigonometric functions on restricted domains.

We can control the precision of $R_{k}$ and $R_{k}^{\mathrm{c}},{ }^{1}$ but a better way to proceed with this will be given in (4) below. The intuition behind using expansions like (2) for approximations (in applications like resampling) is that it is the higher-order terms in the remainder that create problems with fat-tailed distributions, whereas bounding and omitting $R_{k}^{\mathrm{c}}$ will get around such difficulties. Furthermore, the conditions that lead to the sample mean being a consistent estimator of the population mean will typically also lead to the asymptotically correct coverage by the centered expansions. We shall not dwell on these asymptotic issues in this paper, since our focus is on the expansions themselves. Nevertheless, we will touch briefly on this topic at the end of this section, once the derivations are made.

We have centered the expansion around the mean of $y$, but there is a more attractive expansion if we are willing to introduce complex numbers and use bounding results from the theory of characteristic functions. Let $-1 \equiv \mathrm{i}^{2}$ and $(y-\mathrm{E} y) / m \equiv \zeta+2 \pi i_{y}$, where $i_{y} \in \mathbb{Z}, m \in \mathbb{N}$, and $\zeta \in(-\pi, \pi]$, then write

$$
x=\mathrm{e}^{\mathrm{E} y} \mathrm{e}^{2 \pi m i_{y}}\left(\exp \left(\frac{\zeta}{\mathrm{i}}\right)\right)^{\mathrm{i} m} \equiv \mathrm{e}^{\mathrm{E} y} \mathrm{e}^{2 \pi m i_{y}}\left(\xi_{k}+\varrho_{x, k}\right)^{\mathrm{i} m}
$$

where $\xi_{k}:=\sum_{j=0}^{k} \zeta^{j} /\left(\mathrm{i}^{j} j !\right)$. Notice that $i_{y}$ is random, but $m$ is deterministic and to be chosen later. We can now state the bound

$$
\left|\varrho_{x, k}\right| \equiv\left|\mathrm{e}^{\mathrm{i} t}-\sum_{j=0}^{k} \frac{(\mathrm{i} t)^{j}}{j !}\right| \leq \frac{|t|^{k+1}}{(k+1) !}
$$

\footnotetext{
${ }^{1}$ Of course, $R_{k}$ and $R_{k}^{\mathrm{c}}$ can be calculated exactly in theory, but their moments could be empirically problematic (e.g. with fat-tailed densities), hence our approach to bound them instead.
} 
for any $t \in \mathbb{R}$. This is helpful when taking expectations, as it controls the precision by calculating the moment of the next power. A binomial expansion of (4) gives

$$
x=\mathrm{e}^{\mathrm{E} y} \mathrm{e}^{2 \pi m i_{y}} \operatorname{Re}\left(\xi_{k}^{\mathrm{i} m}\right)+R_{x, k}^{\mathrm{c}}, \quad R_{x, k}^{\mathrm{c}}=O_{p}\left(\zeta^{k+1}\right) .
$$

More precisely,

$$
\begin{aligned}
\left|R_{x, k}^{\mathrm{c}}\right| & =\mathrm{e}^{\mathrm{E} y} \mathrm{e}^{2 \pi m i_{y}}\left|\operatorname{Re}\left(\left(\xi_{k}+\varrho_{x, k}\right)^{\mathrm{i} m}-\xi_{k}^{\mathrm{i} m}\right)\right| \\
& \leq \mathrm{e}^{\mathrm{E} y} \mathrm{e}^{2 \pi m i_{y}}\left|\left(\xi_{k}+\varrho_{x, k}\right)^{\mathrm{i} m}-\xi_{k}^{\mathrm{i} m}\right|=\mathrm{e}^{\mathrm{E} y} \mathrm{e}^{2 \pi m i_{y}}\left|\xi_{k}^{\mathrm{i} m}\right|\left|\left(1+\varrho_{x, k} / \xi_{k}\right)^{\mathrm{i} m}-1\right| .
\end{aligned}
$$

Following from the representation of $x$ in terms of a power series in $y$, the form $\mid\left(1+\varrho_{x, k} / \xi_{k}\right)^{\mathrm{i} m}-$ $1 \mid$ is common to all the expansion mentioned in the previous paragraphs. If we can bound it accurately, then we can evaluate these remainders satisfactorily. We therefore present the following results.

Proposition 1 Define the real-valued function $h$ of the complex $\psi$,

$$
h(\psi):=\left|(1+\psi)^{\mathrm{i} m}-1\right|, \quad \text { with } \arg (\psi) \in[-\pi, \pi) .
$$

Then, the global maximum of the function is attained at $h\left(\psi_{\mathrm{m}}\right)=1+\mathrm{e}^{m \pi}$ by $\psi_{\mathrm{m}}=-1-$ $\mathrm{e}^{(2 j+1) \pi / m}$ in the clockwise direction $\left(\arg \left(\psi_{\mathrm{m}}\right)=-\pi\right)$ with $j \in \mathbb{Z}$.

Proof. For any complex $\psi:=a+\mathrm{i} b$, with $a, b$ real and $\theta:=\arg (1+\psi) \in[-\pi, \pi)$, we have

$$
(1+\psi)^{\mathrm{i} m}=|1+\psi|^{\mathrm{i} m} \exp (-m \theta)
$$

where $|1+\psi|^{\mathrm{i} m}=\mathrm{e}^{\mathrm{i} m \log |1+\psi|}$ has modulus 1. Hence,

$$
\begin{aligned}
h(\psi) & =\left|(1+a+b \mathrm{i})^{\mathrm{i} m}-1\right|=\left|\exp \left(\mathrm{i} m \log \sqrt{(1+a)^{2}+b^{2}}-m \theta\right)-1\right| \\
& =|\exp (\mathrm{i} m \log |(1+a) \sec \theta|-m \theta)-1|
\end{aligned}
$$

by $b=(1+a) \tan \theta$, and

$$
\begin{aligned}
h(\psi)^{2} & =\left|\mathrm{e}^{-m \theta} \cos (m \log |(1+a) \sec \theta|)+\mathrm{ie}^{-m \theta} \sin (m \log |(1+a) \sec \theta|)-1\right|^{2} \\
& =\left(\mathrm{e}^{-m \theta} \cos (m \log |(1+a) \sec \theta|)-1\right)^{2}+\mathrm{e}^{-2 m \theta} \sin ^{2}(m \log |(1+a) \sec \theta|) \\
& =1-2 \mathrm{e}^{-m \theta} \cos (m \log |(1+a) \sec \theta|)+\mathrm{e}^{-2 m \theta} .
\end{aligned}
$$


Optimizing $h(\psi)^{2}$ with respect to $a$ gives the first-order condition

$$
\sin \left(m \log \left|\frac{1+a}{\cos \theta}\right|\right)=0
$$

yielding the concentrated

$$
h(\psi)^{2}=1 \mp 2 \mathrm{e}^{-m \theta}+\mathrm{e}^{-2 m \theta}
$$

which is maximized by the corner solution $\theta=-\pi$ and by

$$
\cos \left(m \log \left|\frac{1+a}{\cos \theta}\right|\right)=-1
$$

Hence, with $j \in \mathbb{Z}$, the solution for $a$ can be written as

$$
\log \left|\frac{1+a}{\cos (-\pi)}\right|=(2 j+1) \pi / m
$$

or $|1+a|=\exp ((2 j+1) \pi / m)$. Since $\theta=\arg (1+a+b \mathrm{i})=-\pi$, we have that $1+a<0$ hence

$$
a=-1-\exp ((2 j+1) \pi / m)
$$

and the result follows.

Notice that the triangle inequality gives

$$
\left|(1+\psi)^{\mathrm{i} m}-1\right| \leq\left|(1+\psi)^{\mathrm{i} m}\right|+1=\exp (-m \theta)+1 \leq 1+\exp (m \pi),
$$

where the equality follows from (8). The upper bound of $1+\exp (m \pi)$ is indeed achieved and the proposition tells us which values of $\psi$ achieve it.

By choosing large $m$ or large negative $j$, the solution $\psi_{\mathrm{m}}=-1-\exp ((2 j+1) \pi / m)$ can be made sufficiently close to -1 for most practical purposes. For $|\psi| \leq 1$, the next proposition provides a pointwise logarithmic bound for $h(\psi)$.

Proposition 2 Define the real-valued function $h_{1}$ of the complex $\psi$,

$$
h_{1}(\psi):=\left|(1+\psi)^{\mathrm{i} m}-1\right|-c_{m} \log (1+|\psi|), \quad \text { with } \arg (\psi) \in[-\pi, \pi) \text { and }|\psi| \leq 1 \text {. }
$$

Then, $h_{1}(\psi) \leq 0$ when $c_{m}=\left(1+\mathrm{e}^{m \pi}\right) / \log 2$, with $h_{1}(\psi)=0$ at $\psi=0$. 
Proof. This follows from the previous proposition and the fact that $\log (1+|\psi|)$ is monotonic increasing in $|\psi|$.

As a result of the previous two propositions, the simple bound

$$
\left|(1+\psi)^{\mathrm{i} m}-1\right| \leq\left(1+\mathrm{e}^{m \pi}\right) \min \left(\frac{\log (1+|\psi|)}{\log 2}, 1\right)
$$

applies. The same idea can be applied to any monotonic increasing function of $|\psi|$ (the choice of log was due to its slow variation), however a sharper $|\psi|$-pointwise bound is obtained by the following.

Proposition 3 Define the real-valued function $h$ of the complex $\psi$,

$$
h(\psi):=\left|(1+\psi)^{\mathrm{im}}-1\right|, \quad \text { with } \arg (\psi) \in[-\pi, \pi) \text { and }|\psi| \leq\left|\psi_{0}\right| \in[0,1] .
$$

Then, the maximum of the function is monotonic increasing in $\left|\psi_{0}\right|$ and so is the bound

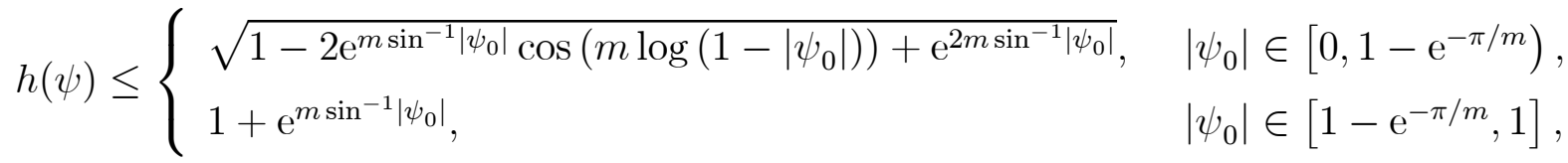

for any given $\left|\psi_{0}\right|$.

Proof. We maximize $h(\psi)^{2}$ as in Proposition 1, but this time subject to the additional condition that $|\psi| \leq\left|\psi_{0}\right|$. However, now $1+a \geq 0$ since $|a| \leq\left|\psi_{0}\right| \leq 1$, and the optimal solution will satisfy $\theta \in[-\pi / 2,0]$ and $a \leq 0$. Visualize the solution as the intersection point (in the lower half plane) of a ray of angle $\theta$ from the origin with a circle of radius $\left|\psi_{0}\right|$ centered around 1. This optimization is easiest to do in terms of $|\psi|$ and $\theta$. To this end, using the definitions $|\psi|^{2}=a^{2}+b^{2}$ and $b^{2}=(1+a)^{2} \tan ^{2} \theta$ gives a quadratic equation for $a$ whose solutions are

$$
a=-\sin ^{2} \theta \pm \sqrt{|\psi|^{2} \cos ^{2} \theta-\sin ^{2} \theta \cos ^{2} \theta}
$$

For $a \leq 0$, the top solution $(+\sqrt{ })$ requires $|\psi|^{2} \in\left[\sin ^{2} \theta, \tan ^{2} \theta\right]$ and the bottom $(-\sqrt{ })$ just $|\psi|^{2} \geq \sin ^{2} \theta$. For $a \in[-|\psi|, 0]$, we need further that

$$
\pm \sqrt{|\psi|^{2} \cos ^{2} \theta-\sin ^{2} \theta \cos ^{2} \theta} \geq \sin ^{2} \theta-|\psi|
$$


Now $\sin ^{2} \theta \leq|\psi|^{2} \leq|\psi|$ since $|\psi| \leq 1$, so the RHS is nonpositive: the top restriction always holds and the bottom one requires $-\sin ^{2} \theta(|\psi|-1)^{2} \leq 0$ which always holds. As a result, $a \in[-|\psi|, 0]$ imposes no further restrictions. In either case, the objective function is

$$
\begin{aligned}
h(\psi)^{2} & =1-2 \mathrm{e}^{-m \theta} \cos (m \log |(1+a) \sec \theta|)+\mathrm{e}^{-2 m \theta} \\
& =1-2 \mathrm{e}^{-m \theta} \cos \left(m \log \left(\cos \theta \pm \sqrt{|\psi|^{2}-\sin ^{2} \theta}\right)\right)+\mathrm{e}^{-2 m \theta}
\end{aligned}
$$

since $1+a \geq 0$ and $\cos \theta \geq 0$. This is a function of $\varphi:=|\psi|^{2}$ and $\theta$, which is to be optimized subject to $\varphi \leq\left|\psi_{0}\right|^{2}$. The augmented function is

$$
1-2 \mathrm{e}^{-m \theta} \cos \left(m \log \left(\cos \theta \pm \sqrt{\varphi-\sin ^{2} \theta}\right)\right)+\mathrm{e}^{-2 m \theta}-l\left(\left|\psi_{0}\right|^{2}-\varphi\right),
$$

leading to the Kuhn-Tucker conditions

$$
l=-\mathrm{e}^{-m \theta} \frac{m \sin \left(m \log \left(\cos \theta \pm \sqrt{\varphi-\sin ^{2} \theta}\right)\right)}{ \pm \sqrt{\varphi-\sin ^{2} \theta}\left(\cos \theta \pm \sqrt{\varphi-\sin ^{2} \theta}\right)} \leq 0, \quad l\left(\left|\psi_{0}\right|^{2}-\varphi\right)=0
$$

and

$$
\cos \left(m \log \left(\cos \theta \pm \sqrt{\varphi-\sin ^{2} \theta}\right)\right) \mp \frac{\sin \theta \sin \left(m \log \left(\cos \theta \pm \sqrt{\varphi-\sin ^{2} \theta}\right)\right)}{\sqrt{\varphi-\sin ^{2} \theta}}=\mathrm{e}^{-m \theta} .
$$

If $l=0$, the last equation becomes $1=\mathrm{e}^{-m \theta}$, hence $\theta=0$ which does not lead to a maximum when plugged into $h$. Hence, $l \neq 0$ and the constraint $|\psi|=\left|\psi_{0}\right|$ is binding, which implies the monotonicity of $h$ in $\left|\psi_{0}\right|$.

Since $l \neq 0$ for the optimum, $\sin (m \log ) \neq 0$ implying that $\cos (m \log ) \neq 1$ unlike in Proposition 1. The objective function cannot be simplified like before, and the first-order condition on $\theta$ seems intractable. We resort instead to bounding the components of $h$. The exponentials' argument is bounded by $\left|\psi_{0}\right|^{2} \geq \sin ^{2} \theta$, hence $-\theta \leq \sin ^{-1}\left|\psi_{0}\right|$. As for the remaining component of $h$, consider the transformation $s= \pm \sqrt{\varphi-\sin ^{2} \theta}$ hence

$$
\theta=-\sin ^{-1} \sqrt{\varphi-s^{2}}=-\cos ^{-1} \sqrt{1-\varphi+s^{2}}
$$

and

$$
\begin{aligned}
h(\psi)^{2} & =1-2 \mathrm{e}^{m \sin ^{-1} \sqrt{\varphi-s^{2}}} \cos \left(m \log \left(\cos \sin ^{-1} \sqrt{\varphi-s^{2}}+s\right)\right)+\mathrm{e}^{2 m \sin ^{-1} \sqrt{\varphi-s^{2}}} \\
& =1-2 \mathrm{e}^{m \cos ^{-1} \sqrt{1-\varphi+s^{2}}} \cos \left(m \log \left(\sqrt{1-\varphi+s^{2}}+s\right)\right)+\mathrm{e}^{2 m \cos ^{-1} \sqrt{1-\varphi+s^{2}}},
\end{aligned}
$$


where the sign of $s$ affects only the logarithmic component. Maximizing $-\cos \left(m \log \left(\sqrt{1-\varphi+s^{2}}+\right.\right.$ $s)$ ) subject to $s \in[-\sqrt{\varphi}, \sqrt{\varphi}]$ gives an interior solution of +1 when

$$
\varphi \in\left[\left(\mathrm{e}^{(2 j+1) \pi / m}-1\right)^{2},\left(\mathrm{e}^{(2 j+1) \pi / m}+1\right)^{2}\right]
$$

where the upper bound is always bigger than 1 but the lower bound is minimized (for the interval to cover all interior solutions) by choosing $j=-1$ for any given $m$, and this latter bound is

$$
\varphi=\left(\mathrm{e}^{-\pi / m}-1\right)^{2}
$$

or $\left|\psi_{0}\right|=1-\mathrm{e}^{-\pi / m}$, giving the solution

$$
s=\frac{-1+\varphi+\mathrm{e}^{-2 \pi / m}}{2 \mathrm{e}^{-\pi / m}}=\frac{\varphi}{2} \mathrm{e}^{\pi / m}+\sinh (-\pi / m)
$$

and $-\cos \left(\log \left(\sqrt{1-\varphi+s^{2}}+s\right)\right)=+1$, hence the monotonic bound

$$
h(\psi)^{2} \leq 1+2 \mathrm{e}^{m \sin ^{-1}\left|\psi_{0}\right|}+\mathrm{e}^{2 m \sin ^{-1}\left|\psi_{0}\right|}=\left(1+\mathrm{e}^{m \sin ^{-1}\left|\psi_{0}\right|}\right)^{2} .
$$

Otherwise, with $-\cos (m \log \cdot)<1$, the largest corner solution is obtained when $s=$ $-\sqrt{\varphi}<0$ (the bottom solution for $a$ ) and

$$
-\cos \left(m \log \left(\sqrt{1-\varphi+s^{2}}+s\right)\right)=-\cos (m \log (1-\sqrt{\varphi}))
$$

hence

$$
h(\psi)^{2} \leq 1-2 \mathrm{e}^{m \sin ^{-1}\left|\psi_{0}\right|} \cos \left(m \log \left(1-\left|\psi_{0}\right|\right)\right)+\mathrm{e}^{2 m \sin ^{-1}\left|\psi_{0}\right|}
$$

and the two bounds on $h$ coincide at the switching point $\left|\psi_{0}\right|=1-\mathrm{e}^{-\pi / m}$. The monotonicity of this bound follows by differentiating then solving for the zeros shows that there are none in $\left(0,1-\mathrm{e}^{-\pi / m}\right)$.

As a result of this proposition and the first one, when $\arg (\psi) \in[-\pi, \pi)$ we have the bound

$$
\left|(1+\psi)^{\mathrm{i} m}-1\right| \leq H(|\psi|),
$$


where

(9)

$$
H(|\psi|):= \begin{cases}\sqrt{1-2 \mathrm{e}^{m \sin ^{-1}|\psi|} \cos (m \log (1-|\psi|))+\mathrm{e}^{2 m \sin ^{-1}|\psi|},}, & |\psi| \in\left[0,1-\mathrm{e}^{-\pi / m}\right), \\ 1+\mathrm{e}^{m \sin ^{-1}|\psi|}, & |\psi| \in\left[1-\mathrm{e}^{-\pi / m}, 1\right], \\ 1+\mathrm{e}^{m \pi}, & \text { otherwise. }\end{cases}
$$

Given the monotonicity property in Proposition 3 and (9), we can work out the bound in (7) as

$$
\left|R_{x, k}^{\mathrm{c}}\right| \leq \mathrm{e}^{\mathrm{E} y} \mathrm{e}^{2 \pi m i_{y}}\left|\xi_{k}^{\mathrm{i} m}\right| H\left(\frac{|\zeta|^{k+1}}{(k+1) !\left|\xi_{k}\right|}\right)
$$

by (5). For calculating expectations, the error for $\mu:=\mathrm{E}(x)$ is denoted by $R_{\mu, k}^{\mathrm{c}}$ and is bounded by

$$
\begin{aligned}
\left|R_{\mu, k}^{\mathrm{c}}\right| & =\mathrm{e}^{\mathrm{E} y}\left|\mathrm{E}\left[\mathrm{e}^{2 \pi m i_{y}} \operatorname{Re}\left(\left(\xi_{k}+\varrho_{x, k}\right)^{\mathrm{i} m}-\xi_{k}^{\mathrm{i} m}\right)\right]\right| \\
& \leq \mathrm{e}^{\mathrm{E} y} \mathrm{E}\left|\mathrm{e}^{2 \pi m i_{y}} \operatorname{Re}\left(\left(\xi_{k}+\varrho_{x, k}\right)^{\mathrm{i} m}-\xi_{k}^{\mathrm{i} m}\right)\right| \\
& \leq \mathrm{e}^{\mathrm{E} y} \mathrm{E}\left[\mathrm{e}^{2 \pi m i_{y}}\left|\xi_{k}^{\mathrm{i} m}\right| H\left(\frac{|\zeta|^{k+1}}{(k+1) !\left|\xi_{k}\right|}\right)\right] .
\end{aligned}
$$

Note the alternative formulation

$$
\left|\xi_{k}^{\mathrm{i} m}\right|=\left.|| \xi_{k}\right|^{\mathrm{i} m}\left|\mathrm{e}^{-m \arg \left(\xi_{k}\right)}=\right| \mathrm{e}^{\mathrm{i} m \log \left|\xi_{k}\right|} \mid \mathrm{e}^{-m \arg \left(\xi_{k}\right)}=\mathrm{e}^{-m \arg \left(\xi_{k}\right)}
$$

which does not contain imaginary quantities.

We conclude this section by analyzing the choice of the deterministic $m$. Recall that $(y-\mathrm{E} y) / m \equiv \zeta+2 \pi i_{y}$, which was introduced before (4). This means that $m$ acts like a scaling integer. Choosing a large $m$ leads to the consistency-like behaviour we mentioned at the outset, here in the sense that the scale shrinks the variate around $\mathrm{E}(y)$ and fewer terms (smaller $k$ ) are needed for the expansion to be accurate. In practical applications, such as the resampling example mentioned earlier, there will be a tradeoff between reducing the magnitude of the remainder terms (requires larger $m$ ) and the imprecision it introduces in the evaluation of the required moments empirically. 


\section{Illustration of the $k$-term expansion and bound's accuracy for $\mathrm{E}(x)$}

We illustrate the performance of the $k$-term expansion and bound given in $(6)$ for $\mathrm{E}(x)$ using two distributions: the normal $y \sim \mathrm{N}(1,1)$ and the gamma $y \sim \operatorname{Gam}(\nu, \lambda)$. In the latter case, the density of the log-gamma $x:=\mathrm{e}^{y} \in(1, \infty)$ is

$$
f_{x}(u)=\frac{\lambda^{\nu}(\log u)^{\nu-1}}{\Gamma(\nu) u^{\lambda+1}} \quad(\nu, \lambda>0),
$$

hence being in the domain of attraction of stable laws with exponent (index) $\lambda$ when $\lambda<2$ : its variance does not exist for $1<\lambda<2$ but its mean does.

Tables 1-15 display the $k$-term expectation, denoted $\mathrm{E}_{k}$, and the bound for the remainder using Monte Carlo methods with $10^{5}$ drawings (and same seed) from the above distributions. The precision of the $k$-term expansion is measured by the ratio $\mathrm{E}_{k} / \mathrm{E}^{*}(x)$, where $\mathrm{E}^{*}(x)$ is the Monte Carlo estimate of $\mathrm{E}(x)$. Each table contains the results for the raw and the centered expansions for $k=2,3,4$ and $m=1,10,50,100,500$ and 1,000.

The tables show that even in the fattest-tailed case of small $\lambda$ and large $\nu$, the performance is very good. Even the 2-term expansion $(k=2)$ is accurate, especially when we choose $m$ not too small. All the tables indicate that choosing a large $m$ increases the accuracy of both the expansion and bound. Centered expansion are, on the whole, more accurate when we take $m>1$. The case of the well behaved log-normal stands in contrast to the case of the fat-tailed log-gamma whose variance does not exist.

Unreported calculations show that the expansions we derive using complex numbers are vastly more accurate than the ones that do not use them like the introductory (1)-(2). To illustrate this point, consider $y=10$, then $x:=\mathrm{e}^{y}=22,026.4657$. First, consider the raw expansion (1) with no complex numbers or $m$ involved. The 2-term expansion gives only $x \approx 61$. We need a 30-term expansion to obtain a good approximation of $x$.

Second, we consider (6) for $m=1$ and different values of $k$. The 2-term expansion gives $9,447.5$ and the corresponding bound for the remainder (10) is 83,879 . However, here we only need a 12-term expansion to obtain a good approximation of $x$ with a bound of 24.83 . 
Finally, we consider the same expansion as in (6) for fixed $k=2$ and different values of $m$. For $m=100$ the expansion is 22,396 while the bound is 532 . Taking $m=10,000$, the 2-term expansion is extremely accurate and the bound for the remainder is very precise, namely 0.0519 .

\section{Concluding comments}

Using complex analysis, we provided expansions and bounds for the expectation of a variate $x$ in terms of the moments of a transformation $y$ of $x$. We then illustrated the accuracy of the expansions and bounds by simulating distributions, including ones whose higher order moments do not exist. The results are very good and show that the applications cited in the introduction would benefit from our formulae.

In this paper, we assumed $g$ to be invertible because we dealt with $x \equiv g\left(g^{-1}(x)\right)$ and expanded a chosen $g$ that converges fast, the motivating reason being that we wanted an accurate expansion for $x$ and its expectation. This invertibility of $g$ is not required for our propositions to apply. They are general and can be used directly in the case of any function $x=g(z)$ that is not necessarily invertible (e.g. by a Taylor expansion of $g(z)^{-\mathrm{i}}$, or even the composition $x=g_{1}\left(g_{2}(z)\right)$ where we would expand $g_{1}$ only. If so, the only required alteration would not be in our propositions, but in the coefficients of the expansion preceding Proposition 1. To illustrate with the Box-Cox transformation

$$
z:=\frac{x^{p}-1}{p}
$$

if we are interested in the expectation of the original variate $x$, omitting the centring for ease of exposition gives

$$
x=(1+p z)^{1 / p}=\left(\mathrm{e}^{(\mathrm{i} p)^{-1} \log (1+p z)}\right)^{\mathrm{i}}=\left(\xi_{k}+\varrho_{x, k}\right)^{\mathrm{i}}, \quad \text { with } \xi_{k}:=\sum_{j=0}^{k} \frac{(\log (1+p z))^{j}}{(\mathrm{i} p)^{j} j !}
$$

and the same propositions apply as before. It is also possible to expand by something other than an exponential function, as discussed in Section 2 , or simply expand $(1+p z)^{-\mathrm{i} / p}$ by 
the binomial (relevant special case of a Taylor series) but the latter's convergence would be conditional.

\section{REFERENCES}

Abadir, K. M. (1999). An introduction to hypergeometric functions for economists. Econometric Reviews, 18, 287-330.

Abadir, K. M. and J. R. Magnus (2002). Notation in econometrics: a proposal for a standard. Econometrics Journal, 5, 76-90.

Abadir, K. M. and G. Talmain (2005). Autocovariance functions of series and of their transforms. Journal of Econometrics, 124, 227-252.

Athreya, K. B. (1987). Bootstrap of the mean in the infinite variance case. Annals of Statistics, 15, 724-731.

Backus, D., M. Chernov and I. Martin (2011). Disasters implied by equity index options. Journal of Finance, forthcoming.

Knight, K. (1989). On the bootstrap of the mean in the infinite variance case. Annals of Statistics, 17, 1168-1175.

Martin, I. W. R. (2008). Disasters and the welfare cost of uncertainty. American Economic Review, 98, 74-78.

Politis, D. N., J. P. Romano and M. Wolf (1999). Subsampling. Springer-Verlag, New York.

Taylor, J. M. G. (1986). The retransformed mean after a fitted power transformation. Journal of the American Statistical Association, 81, 114-118.

Whittaker, E. T. and G. N. Watson (1997). A course of modern analysis, 4th edition. Cambridge University Press, Cambridge. 
Table 1: $y \sim \operatorname{Gam}(2,1.1), k=2, \mathrm{E}^{*}(x)=48.113837381$

\begin{tabular}{ccccccc}
\hline \hline & \multicolumn{3}{c}{ noncentered } & \multicolumn{3}{c}{ centered } \\
\hline$m$ & $\mathrm{E}_{k}$ & Bound & $\mathrm{E}_{k} / \mathrm{E}^{*}(x)$ & $\mathrm{E}_{k}$ & Bound & $\mathrm{E}_{k} / \mathrm{E}^{*}(x)$ \\
\hline 1 & 36.02031518 & 79.52389775 & 0.748647731 & 31.85063757 & 112.8084388 & 0.661984978 \\
10 & 22.75139093 & 739.1814387 & 0.472865857 & 59.81391499 & 232.8180046 & 1.243174900 \\
50 & 50.59838790 & 3.901968792 & 1.051639001 & 49.48870901 & 2.079265927 & 1.028575389 \\
100 & 48.72227366 & 0.882972177 & 1.012645765 & 48.45340538 & 0.488301544 & 1.007057595 \\
500 & 48.13800244 & 0.034209684 & 1.000502248 & 48.12736420 & 0.019144562 & 1.000281142 \\
1000 & 48.11987728 & 0.008543904 & 1.000125534 & 48.11721864 & 0.004783143 & 1.000070276
\end{tabular}

Yu, J., Z. Yang and X. Zhang (2006). A class of nonlinear stochastic volatility models and its implications for pricing currency options. Computational Statistics 85 Data Analysis, 51, 2218-2231.

Table 2: $y \sim \operatorname{Gam}(2,1.1), k=3, \mathrm{E}^{*}(x)=48.113837381$

\begin{tabular}{ccccccc}
\hline \hline \multicolumn{3}{c}{ noncentered } & \multicolumn{3}{c}{ centered } \\
\hline$m$ & $\mathrm{E}_{k}$ & Bound & $\mathrm{E}_{k} / \mathrm{E}^{*}(x)$ & $\mathrm{E}_{k}$ & Bound & $\mathrm{E}_{k} / \mathrm{E}^{*}(x)$ \\
\hline 1 & 686.7873982 & 4271.121409 & 14.27421789 & 1295.665613 & 9251.947117 & 26.92916806 \\
10 & 62.26001429 & 66.30685719 & 1.29401473 & 53.4084961 & 18.29835898 & 1.110044407 \\
50 & 48.13614219 & 0.183205259 & 1.000463584 & 48.12306332 & 0.087691534 & 1.000191752 \\
100 & 48.11525298 & 0.022820961 & 1.000029422 & 48.11441994 & 0.010941749 & 1.000012108 \\
500 & 48.11383966 & 0.000182483 & 1.000000047 & 48.11383832 & $8.74994 \mathrm{E}-05$ & 1.000000019
\end{tabular}


Table 3: $y \sim \operatorname{Gam}(2,1.1), k=4, \mathrm{E}^{*}(x)=48.113837381$

\begin{tabular}{ccccccc}
\hline \hline & \multicolumn{3}{c}{ noncentered } & \multicolumn{3}{c}{ centered } \\
\hline$m$ & $\mathrm{E}_{k}$ & Bound & $\mathrm{E}_{k} / \mathrm{E}^{*}(x)$ & $\mathrm{E}_{k}$ & Bound & $\mathrm{E}_{k} / \mathrm{E}^{*}(x)$ \\
\hline 1 & 656.6211587 & 17041.23391 & 13.64724151 & 1280.201915 & 34238.65768 & 26.60776992 \\
10 & 45.93426141 & 5.088503652 & 0.954699602 & 47.10456151 & 2.057393128 & 0.979023168 \\
50 & 48.10824978 & 0.008049875 & 0.999883867 & 48.11153053 & 0.003306909 & 0.999952054 \\
100 & 48.11348322 & 0.000503168 & 0.999992639 & 48.11369172 & 0.000206678 & 0.999996973
\end{tabular}

Table 4: $y \sim \operatorname{Gam}(2,1.3), k=2, \mathrm{E}^{*}(x)=16.2588462282$

\begin{tabular}{ccccccc}
\hline \hline & \multicolumn{3}{c}{ noncentered } & \multicolumn{3}{c}{ centered } \\
\hline$m$ & $\mathrm{E}_{k}$ & Bound & $\mathrm{E}_{k} / \mathrm{E}^{*}(x)$ & $\mathrm{E}_{k}$ & Bound & $\mathrm{E}_{k} / \mathrm{E}^{*}(x)$ \\
\hline 1 & 10.90027129 & 39.49266418 & 0.67042096 & 12.04682277 & 28.36346446 & 0.740939585 \\
10 & 20.05461030 & 42.98010744 & 1.233458391 & 19.51817111 & 14.75765738 & 1.200464709 \\
50 & 16.56024987 & 0.450947284 & 1.018537825 & 16.41722038 & 0.232551018 & 1.009740799 \\
100 & 16.33345748 & 0.106994115 & 1.004588963 & 16.29821230 & 0.056215388 & 1.002421210 \\
500 & 16.26182072 & 0.004208903 & 1.000182946 & 16.26041783 & 0.002224563 & 1.000096661 \\
1000 & 16.25958977 & 0.001051677 & 1.000045732 & 16.25923910 & 0.000555951 & 1.000024164
\end{tabular}

Table 5: $y \sim \operatorname{Gam}(2,1.3), k=3, \mathrm{E}^{*}(x)=16.2588462282$

\begin{tabular}{ccccccc}
\hline \hline \multicolumn{3}{c}{ noncentered } & \multicolumn{3}{c}{ centered } \\
\hline$m$ & $\mathrm{E}_{k}$ & Bound & $\mathrm{E}_{k} / \mathrm{E}^{*}(x)$ & $\mathrm{E}_{k}$ & Bound & $\mathrm{E}_{k} / \mathrm{E}^{*}(x)$ \\
\hline 1 & 357.3868597 & 2437.040583 & 21.98107139 & 179.4132451 & 1207.692768 & 11.03480792 \\
10 & 17.27127102 & 3.435483172 & 1.062269166 & 16.65696979 & 1.263222364 & 1.024486582 \\
50 & 16.26061542 & 0.017805411 & 1.000108814 & 16.25954838 & 0.008141218 & 1.000043186 \\
100 & 16.25895783 & 0.002222322 & 1.000006864 & 16.25889039 & 0.001016834 & 1.000002716 \\
500 & 16.25884641 & $1.77665 \mathrm{E}-05$ & 1.000000011 & 16.2588463 & $8.12314 \mathrm{E}-06$ & 1.000000004
\end{tabular}


Table 6: $y \sim \operatorname{Gam}(2,1.3), k=4, \mathrm{E}^{*}(x)=16.2588462282$

\begin{tabular}{ccccccc}
\hline \hline \multicolumn{3}{c}{ noncentered } & \multicolumn{3}{c}{ centered } \\
\hline$m$ & $\mathrm{E}_{k}$ & Bound & $\mathrm{E}_{k} / \mathrm{E}^{*}(x)$ & $\mathrm{E}_{k}$ & Bound & $\mathrm{E}_{k} / \mathrm{E}^{*}(x)$ \\
\hline 1 & 347.0041084 & 9138.275446 & 21.34248049 & 175.6001415 & 4476.162379 & 10.80028306 \\
10 & 16.05949307 & 0.393932431 & 0.987738788 & 16.17271909 & 0.155668905 & 0.994702752 \\
50 & 16.25840397 & 0.000633313 & 0.999972799 & 16.25867084 & 0.000250341 & 0.999989213 \\
100 & 16.25881833 & $3.95755 \mathrm{E}-05$ & 0.999998284 & 16.25883519 & $1.56374 \mathrm{E}-05$ & 0.999999321
\end{tabular}

Table 7: $y \sim \operatorname{Gam}(1,1.1), k=2, \mathrm{E}^{*}(x)=7.64132294$

\begin{tabular}{ccccccc}
\hline \hline & \multicolumn{3}{c}{ noncentered } & \multicolumn{3}{c}{ centered } \\
\hline$m$ & $\mathrm{E}_{k}$ & Bound & $\mathrm{E}_{k} / \mathrm{E}^{*}(x)$ & $\mathrm{E}_{k}$ & Bound & $\mathrm{E}_{k} / \mathrm{E}^{*}(x)$ \\
\hline 1 & 5.348890454 & 18.11267799 & 0.69999534 & 5.547758808 & 13.9716725 & 0.726020723 \\
10 & 9.070487448 & 21.76358109 & 1.18703103 & 9.250433075 & 11.70554432 & 1.210580045 \\
50 & 7.779876421 & 0.208442261 & 1.018132132 & 7.738604908 & 0.144474011 & 1.012731037 \\
100 & 7.675580602 & 0.049193328 & 1.00448321 & 7.665445087 & 0.034536134 & 1.003156802 \\
500 & 7.642688123 & 0.001931828 & 1.000178657 & 7.642285167 & 0.001361787 & 1.000125924 \\
1000 & 7.641664198 & 0.000482679 & 1.000044659 & 7.641563478 & 0.000340293 & 1.000031478
\end{tabular}

Table 8: $y \sim \operatorname{Gam}(1,1.1), k=3, \mathrm{E}^{*}(x)=7.64132294$

\begin{tabular}{ccccccc}
\hline \hline \multicolumn{3}{c}{ noncentered } & \multicolumn{3}{c}{ centered } \\
\hline$m$ & $\mathrm{E}_{k}$ & Bound & $\mathrm{E}_{k} / \mathrm{E}^{*}(x)$ & $\mathrm{E}_{k}$ & Bound & $\mathrm{E}_{k} / \mathrm{E}^{*}(x)$ \\
\hline 1 & 132.5229086 & 1132.385351 & 17.34292734 & 120.6061654 & 847.8742229 & 15.78341424 \\
10 & 8.141174023 & 1.725595451 & 1.065414207 & 7.936898676 & 0.954853041 & 1.038681225 \\
50 & 7.642193478 & 0.008490408 & 1.000113925 & 7.641844525 & 0.005513082 & 1.000068258 \\
100 & 7.641377893 & 0.001059513 & 1.000007191 & 7.641355802 & 0.000688324 & 1.0000043 \\
500 & 7.641323032 & $8.47005 \mathrm{E}-06$ & 1.000000012 & 7.641322996 & $5.50162 \mathrm{E}-06$ & 1.000000007
\end{tabular}


Table 9: $y \sim \operatorname{Gam}(1,1.1), k=4, \mathrm{E}^{*}(x)=7.64132294$

\begin{tabular}{ccccccc}
\hline \hline & \multicolumn{3}{c}{ noncentered } & \multicolumn{3}{c}{ centered } \\
\hline$m$ & $\mathrm{E}_{k}$ & Bound & $\mathrm{E}_{k} / \mathrm{E}^{*}(x)$ & $\mathrm{E}_{k}$ & Bound & $\mathrm{E}_{k} / \mathrm{E}^{*}(x)$ \\
\hline 1 & 137.4851024 & 3506.24111 & 17.99231669 & 116.763729 & 2896.920893 & 15.28056462 \\
10 & 7.544974283 & 0.194066642 & 0.987391102 & 7.580423302 & 0.115830347 & 0.992030223 \\
50 & 7.641105291 & 0.000311884 & 0.999971516 & 7.641192612 & 0.00018637 & 0.999982944 \\
100 & 7.641309205 & $1.94899 \mathrm{E}-05$ & 0.999998202 & 7.64131473 & $1.1645 \mathrm{E}-05$ & 0.999998925
\end{tabular}

Table 10: $y \sim \operatorname{Gam}(1,1.3), k=2, \mathrm{E}^{*}(x)=4.1276252974$

\begin{tabular}{ccccccc}
\hline \hline & \multicolumn{3}{c}{ noncentered } & \multicolumn{3}{c}{ centered } \\
\hline$m$ & $\mathrm{E}_{k}$ & Bound & $\mathrm{E}_{k} / \mathrm{E}^{*}(x)$ & $\mathrm{E}_{k}$ & Bound & $\mathrm{E}_{k} / \mathrm{E}^{*}(x)$ \\
\hline 1 & 3.222377874 & 7.159466827 & 0.780685659 & 3.459858499 & 5.498965123 & 0.838220102 \\
10 & 4.610126678 & 1.932521013 & 1.116895635 & 4.478563404 & 1.101408133 & 1.085021794 \\
50 & 4.150268872 & 0.033086837 & 1.005485860 & 4.142876889 & 0.022165928 & 1.003695004 \\
100 & 4.133258332 & 0.008031035 & 1.001364716 & 4.131424273 & 0.005412930 & 1.000920378 \\
500 & 4.127850246 & 0.000318227 & 1.000054498 & 4.127777070 & 0.000214898 & 1.000036770 \\
1000 & 4.127681532 & $7.95323 \mathrm{E}-05$ & 1.000013624 & 4.127663239 & $5.37101 \mathrm{E}-05$ & 1.000009192
\end{tabular}

Table 11: $y \sim \operatorname{Gam}(1,1.3), k=3, \mathrm{E}^{*}(x)=4.1276252974$

\begin{tabular}{ccccccc}
\hline \hline \multicolumn{3}{c}{ noncentered } & \multicolumn{3}{c}{ centered } \\
\hline$m$ & $\mathrm{E}_{k}$ & Bound & $\mathrm{E}_{k} / \mathrm{E}^{*}(x)$ & $\mathrm{E}_{k}$ & Bound & $\mathrm{E}_{k} / \mathrm{E}^{*}(x)$ \\
\hline 1 & 41.10209787 & 298.1583280 & 9.957807433 & 35.42614118 & 246.8631630 & 8.582693104 \\
10 & 4.179104175 & 0.166211312 & 1.012471790 & 4.157808888 & 0.098258707 & 1.007312580 \\
50 & 4.127715779 & 0.001092510 & 1.000021921 & 4.127678059 & 0.000686532 & 1.000012783 \\
100 & 4.127630986 & 0.000136464 & 1.000001378 & 4.127628611 & $8.57695 \mathrm{E}-05$ & 1.000000803 \\
500 & 4.127625307 & $1.08802 \mathrm{E}-06$ & 1.000000002 & 4.127625303 & $6.83128 \mathrm{E}-07$ & 1.000000001
\end{tabular}


Table 12: $y \sim \operatorname{Gam}(1,1.3), k=4, \mathrm{E}^{*}(x)=4.1276252974$

\begin{tabular}{ccccccc}
\hline \hline \multicolumn{3}{c}{ noncentered } & \multicolumn{3}{c}{ centered } \\
\hline$m$ & $\mathrm{E}_{k}$ & Bound & $\mathrm{E}_{k} / \mathrm{E}^{*}(x)$ & $\mathrm{E}_{k}$ & Bound & $\mathrm{E}_{k} / \mathrm{E}^{*}(x)$ \\
\hline 1 & 41.16945050 & 1034.516608 & 9.974124958 & 35.33930121 & 867.5052936 & 8.561654382 \\
10 & 4.116393816 & 0.020057047 & 0.997278948 & 4.120839821 & 0.011688021 & 0.998356082 \\
50 & 4.127602697 & $3.22434 \mathrm{E}-05$ & 0.999994525 & 4.127612121 & $1.87732 \mathrm{E}-05$ & 0.999996808 \\
100 & 4.127623875 & $2.01233 \mathrm{E}-06$ & 0.999999655 & 4.127624469 & $1.17092 \mathrm{E}-06$ & 0.999999799
\end{tabular}

Table 13: $y \sim \mathrm{N}(1,1), k=2, \mathrm{E}^{*}(x)=4.4703660027$

\begin{tabular}{ccccccc}
\hline \hline & \multicolumn{3}{c}{ noncentered } & \multicolumn{3}{c}{ centered } \\
\hline$m$ & $\mathrm{E}_{k}$ & Bound & $\mathrm{E}_{k} / \mathrm{E}^{*}(x)$ & $\mathrm{E}_{k}$ & Bound & $\mathrm{E}_{k} / \mathrm{E}^{*}(x)$ \\
\hline 1 & 2.98772013 & 11.18380332 & 0.668339042 & 4.098873968 & 3.711456770 & 0.916898967 \\
10 & 4.573313241 & 0.160009011 & 1.023028816 & 4.499708029 & 0.045612240 & 1.006563674 \\
50 & 4.474510726 & 0.005887077 & 1.000927155 & 4.471546512 & 0.001749706 & 1.000264074 \\
100 & 4.471402220 & 0.001467883 & 1.000231797 & 4.470661165 & 0.000436851 & 1.000066026 \\
500 & 4.470407452 & $5.86654 \mathrm{E}-05$ & 1.000009272 & 4.470377810 & $1.74653 \mathrm{E}-05$ & 1.000002641 \\
1000 & 4.470376365 & $1.46655 \mathrm{E}-05$ & 1.000002318 & 4.470368954 & $4.36445 \mathrm{E}-06$ & 1.000000660
\end{tabular}

Table 14: $y \sim \mathrm{N}(1,1), k=3, \mathrm{E}^{*}(x)=4.4703660027$

\begin{tabular}{ccccccc}
\hline \hline \multicolumn{3}{c}{ noncentered } & \multicolumn{3}{c}{ centered } \\
\hline$m$ & $\mathrm{E}_{k}$ & Bound & $\mathrm{E}_{k} / \mathrm{E}^{*}(x)$ & $\mathrm{E}_{k}$ & Bound & $\mathrm{E}_{k} / \mathrm{E}^{*}(x)$ \\
\hline 1 & 49.77001850 & 409.4277092 & 11.13332073 & 11.14246140 & 66.45933835 & 2.492516585 \\
10 & 4.472402982 & 0.011294884 & 1.000455663 & 4.470738257 & 0.002604166 & 1.000083272 \\
50 & 4.470369340 & $8.97957 \mathrm{E}-05$ & 1.000000747 & 4.470366608 & $2.07783 \mathrm{E}-05$ & 1.000000135 \\
100 & 4.470366211 & $1.12231 \mathrm{E}-05$ & 1.000000047 & 4.470366041 & $2.59425 \mathrm{E}-06$ & 1.000000008 \\
500 & 4.470366003 & $8.13632 \mathrm{E}-08$ & 1.000000000 & 4.470366003 & $1.52491 \mathrm{E}-08$ & 1.000000000
\end{tabular}


Table 15: $y \sim \mathrm{N}(1,1), k=4, \mathrm{E}^{*}(x)=4.4703660027$

\begin{tabular}{ccccccc}
\hline \hline & \multicolumn{3}{c}{ noncentered } & \multicolumn{3}{c}{ centered } \\
\hline$m$ & $\mathrm{E}_{k}$ & Bound & $\mathrm{E}_{k} / \mathrm{E}^{*}(x)$ & $\mathrm{E}_{k}$ & Bound & $\mathrm{E}_{k} / \mathrm{E}^{*}(x)$ \\
\hline 1 & 51.04836227 & 1304.891883 & 11.41928026 & 11.12050304 & 190.6877168 & 2.487604601 \\
10 & 4.469867668 & 0.000738275 & 0.999888525 & 4.470274316 & 0.000136150 & 0.999979490 \\
50 & 4.470365169 & $1.17793 \mathrm{E}-06$ & 0.999999813 & 4.470365852 & $2.13008 \mathrm{E}-07$ & 0.999999966 \\
100 & 4.470365951 & $6.65426 \mathrm{E}-08$ & 0.999999988 & 4.470365993 & $9.62026 \mathrm{E}-09$ & 0.999999998
\end{tabular}

